\title{
Seltene, aber häufig übersehene endokrinologische Krankheitsbilder
}

\author{
Georg Benker, Matthias Gruber, Stefan R. Bornstein, Andreas Barthel
}

Was ist neu?

- Familiäre hypokalziurische Hyperkalzämie: Bei der familiären hypokalziurischen Hyperkalziämie (FHH) besteht eine genetisch bedingte „Sollwertverstellung" des in Nebenschilddrüsen- und Tubuluesepithelzellen der Niere exprimierten Calcium-sensing-Rezeptors (CaSR). Im Gegensatz zum primären Hyperparathyreoidismus - von der eine $\mathrm{FHH}$ abzugrenzen ist - resultiert hieraus eine asymptomatische, nicht behandlungsbedürftige Hyperkalzämie.

- Schilddrüsenhormonresistenz: Die eindeutige Abgrenzung einer zentralen Hyperthyreose im Rahmen eines TSHom von einer Schilddrüsenhormonresistenz ist oft schwierig. Neben dem klinischen Befund spielt hier auch die molekulargenetische Diagnostik eine wichtige Rolle, weil mittlerweile spezifische Mutationen identifiziert werden konnten, die kausal mit einer Schilddrüsenhormonresistenz in Zusammenhang stehen.

- Testikuläre adrenale Resttumoren: Bei der Abklärung eines unklaren Hodenbefundes sind testikuläre adrenale Resttumore (TART) eine wichtige Differenzialdiagnose, weil sie von malignen Tumoren abgegrenzt werden müssen. Während TART im Rahmen des adrenogenitalen Syndroms beobachtet werden und ansonsten keiner spezifischen Therapie bedürfen, erfordern maligne Hodentumoren eine umgehende Orchiektomie.
In der Endokrinologie gibt es eine Reihe seltener, aber relevanter Differenzialdiagnosen, die deswegen in Erwägung gezogen werden müssen, weil sich zum Teil weitreichende therapeutische Konsequenzen ergeben können. Alle drei im Folgenden beschriebenen Krankheitsbilder spielen bei der Abklärung häufiger bzw. bedrohlicher Erkrankungen eine wichtige Rolle.

\section{Familiäre hypokalziurische Hyperkalzämie}

Die Serumkonzentration von Kalzium-Ionen wird physiologischerweise innerhalb sehr enger Grenzen gehalten und unterliegt deshalb komplexen Regulationsmechanismen. Auf molekularer Ebene geschieht dies in der Nebenschilddrüse und der Niere (Tubulusepithelien) durch den sogenannten Calcium-Sensing-Rezeptor (CaSR), ein G-Protein gekoppeltes Transmembranprotein. Der CaSR registriert eine Verminderung der Serum-Kalziumkonzentration und stimuliert die Parathormonsekretion. Umgekehrt hemmt der CaSR bei erhöhten Kalziumkonzentrationen die Sekretion von Parathormon. In ähnlicher Weise wird die tubuläre Kalziumrückresorption in der Niere zum Teil über direkte Einflüsse auf den CaSR reguliert [1]

Die Identifikation funktionell relevanter Mutationen im CaSR-Gen hat entscheidend zum Ver- ständnis der Calciumhomöostase und zur Aufklärung der FHH-Pathogenese beigetragen [2]. Mittlerweile wurden viele funktionell relevante Polymorphismen im CaSR-Gen (zytogenetische Lokalisation 3q21.1) beschrieben. Diese als FHH1 bezeichnete und mit Abstand häufigste Form folgt einem autosomal-dominanten Erbgang mit hoher Penetranz. Darüber hinaus wurden Mutationen auf anderen Chromosomen in Genen lokalisiert, die funktionell dem CaSR (z.B. im Bereich der Signaltransduktion des Rezeptors) zugeordnet werden konnten (FHH2: inaktivierende Mutation in einem G-Protein [3]; FHH3: Missense-Mutation in einem Adapterprotein [4]). Der CaSR ist auch ein therapeutischer Angriffspunkt der Calcimimetika (Prototyp Cinacalcet [5]) zur Behandlung therapieresistenter Hyperkalzämien beim primären Hyperparathyreoidismus.

Klinisch findet sich bei der FHH - abhängig vom Genotyp - fast immer eine milde ausgeprägte und deshalb symptomlose Hyperkalzämie. Bei stärker ausgeprägter Hyperkalzämie können jedoch auch typische klinische Erscheinungen auftreten, in Einzelfällen wurden Pankreatitiden beschrieben. Diagnostische Probleme kommen oft dadurch zustande, dass durch die „Sollwertverstellung“ des CaSR bei der FHH oft erhöhte (oder noch normwertige, aber inadäquat hohe) ParathormonSerumkonzentrationen vorgefunden werden, so dass die Abgrenzung vom primären Hyperparathyreoidismus schwierig sein kann [6]. Insbesondere kann dies der Fall sein, wenn aufgrund einer ebenfalls vorliegenden Vitamin-D-Hypovitaminose zusätzlich ein sekundärer Hyperparathyreoidismus besteht, was in unseren Breitengraden sehr häufig der Fall ist [7]. Die Abgrenzung einer FHH insbesondere vom primären Hyperparathyreoidismus ist aber deswegen sehr wichtig, weil beim primären Hyperparathyreoidismus langfristig Organschäden wie z.B. eine Osteoporose durch den erhöhten Knochenabbau oder eine Nephrolithiasis drohen. Letztere beruht auf der erhöhten Kalziumausscheidung im Urin, was ein wichtiges differenzialdiagnostisches Kriterium für die Abgrenzung von der FHH ist, bei der die Kalziumausscheidung im Urin charakteristischerweise oft auffällig niedrig ist. Dieser wegweisende Befund kann durch molekulargenetische Diagnostik des CaSR-Gens weiterverfolgt werden, der Nachweis einer funktionell relevanten Mutation ist schließlich zielführend und bestätigt die Diagnose einer FHH. Da aber bis zu ein Drittel der 
Familien mit FHH keine Mutation im CaSR-Gen haben, ist der Negativbefund nicht beweisend. Das wichtigste Kriterium in unklaren (atypischen) Fällen bleibt die Familienanamnese bzw. die Familienuntersuchung, da die Störung infolge Symptomarmut bzw. -freiheit unerkannt geblieben sein kann.

\section{Klinische Relevanz}

Die familiäre hypokalziurische Hyperkalzämie (FHH) sollte bei der Abklärung einer Hyperkalzämie berücksichtigt werden. Sie muss insbesondere von einem primären Hyperparathyreoidismus abgegrenzt werden. Während letzterer oftmals mittels einer Parathyreoidektomie behandelt wird, ist eine FHH eine harmlose Stoffwechselanomalie, die in der Regel keiner weiteren Behandlung bedarf.

\section{Schilddrüsenhormonresistenz}

In der hausärztlichen Versorgung hat sich ein Screening auf Schilddrüsen-Funktionsstörungen mittels TSH durchgesetzt. Dieses - an sich rationelle - Vorgehen versagt bei Erkrankungen, bei denen das TSH fehlt oder vermindert ist, sowie bei Störungen der Rezeptoren für TSH oder Schilddrüsenhormone. Im ersten Fall wird u.U. eine Hypothyreose maskiert, im zweiten Fall vorgetäuscht.

Ursachen einer Schilddrüsenhormonresistenz sind in etwa $85 \%$ der Fälle Punktmutationen in Bereichen des TRHB-Gens (thyroid hormone receptor beta-Gen), welche die Hormon-bindende Domäne der Schilddrüsenhormon-Rezeptor-BetaIsoform codieren. Die Mutationen sind funktionell relevant, weil sie die Affinität des Rezeptors zum Liganden (T3) beeinträchtigten. Beschrieben wurden bisher weit mehr als 100 Mutationen in familiären Clustern - zumeist mit autosomal-dominantem Erbgang. In ca. 15\% findet man keine Mutationen im TRHB-Gen, sondern in anderen Genen, deren Transkripte eine Rolle spielen bei der Schilddrüsenhormonwirkung (TRHA-Gen), dem Transport von Schilddrüsenhormon in die Zelle (MCT8-Gen) und dem Schilddrüsenhormonmetabolismus (SECISBP2-Gen) [8-10]. Klinisch finden sich bei der Schilddrüsenhormonresistenz - abhängig von der zugrundeliegenden spezifischen Mutation - sehr variable Manifestationen, die insbesondere bei TRHB-Mutationen eher milde sind [11]. Neben einer Struma treten z.B. Tachykardien auf, weiterhin werden unspezifische psychomotorische Störungen (Hyperaktivität, Lernbeeinträchtigungen), Entwicklungsverzögerungen und selten Beeinträchtigungen des Hörvermögens beobachtet. Laborchemisch findet sich die Konstellation eines erhöhten oder inadäquat hohen TSH in Kombination mit einem erhöhten fT3 und/ oder fT4.
Die wichtigste Differenzialdiagnose in einer solchen Situation ist die zentrale Hyperthyreose aufgrund einer autonomen TSH-Produktion. Die Abgrenzung ist häufig schwierig, aber dennoch sehr wichtig: Einer autonomen TSH-Produktion liegt meist ein Hypophysenadenom (TSHom) zugrunde. Im letzteren Fall würde eine neurochirurgische Intervention mit kurativem Ansatz angestrebt. Eine bildgebende Diagnostik der Hypophyse (MRT) hilft hier wegen der geringen Spezifität nicht weiter. Beim TSHom findet sich gelegentlich eine funktionelle Veränderung weiterer hypophysärer Achsen. Außerdem zeigt sich häufig eine vermehrte Sekretion der alpha-Untereinheit des TSH durch die Adenomzellen, was wegweisend für ein TSHom ist. Diagnostisch zielführend für eine Schilddrüsenhormonresistenz ist der Nachweis einer funktionell relevanten Mutation - vor allem durch molekulargenetische Analyse des TRHB-Gens.

\section{Klinische Relevanz}

Bei der Abklärung einer Hyperthyreose ist die Schilddrüsenhormonresistenz eine besondere diagnostische Herausforderung, weil hier eine „Sollwertverstellung“ der Schilddrüsenhormonparameter mit einem erhöhten TSH-Wert und oft relativ milden Symtomen vorliegt. Einer „echten“ TSH-abhängigen zentralen Hyperthyreose liegt zumeist ein TSHom zugrunde, das in der Regel operativ angegangen wird.

\section{Testikuläre adrenale Resttumoren}

Der Begriff des „adrenogenitalen Syndroms“ (AGS) beschreibt Störungen in der Steroidbiosynthese der Nebennieren: Die lebenswichtigen Hormone Cortisol und Aldosteron werden vermindert (Stichwort „Salzverlust-Syndrom“) und Nebennierenandrogene vermehrt gebildet [12]. Schwere homozygote oder compound-heterozygote Defekte sind lebensbedrohlich. Leichte Formen führen evtl. nur zum „late-onset“ AGS mit mehr oder weniger ausgeprägter Virilisierung. In der Praxis ist - leider die Terminologie manchmal unscharf. So werden gelegentlich selbst heterozygote Mutationen (also ein „Überträgerstatus“) als „AGS“ bezeichnet. Die Mutationen betreffen das 21-Hydroxylasegen (häufig) sowie (seltener) das $11 \beta$-Hydroxylaseund das $3 \beta$-Hydroxysteroid-Dehydrogenasegen. Der Schweregrad der Mutation kann zum völligen Funktionsverlust führen (z.B. durch eine Deletion oder ein Stop-Codon) oder zu einer reduzierten Funktion (z.B. die häufige Mutation Val281Leu im 21-Hydroxylase-Gen).

Bei testikulären adrenalen Resttumoren (TART) handelt es sich um ektopes adrenales Gewebe im Bereich der Hoden, das dort im Rahmen eines AGS vorliegt. TART gehören neben den adrenalen Myelolipomen zu den Spätfolgen des AGS - wobei die schweren, klassischen Formen überwiegen.

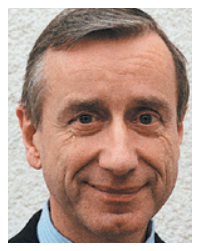

Prof. Dr. Georg Benker ist Facharzt für Innere Medizin und Endokrinologie am Endokrinologikum Ruhr, Bochum georg.benker@ endokrinologikum.com

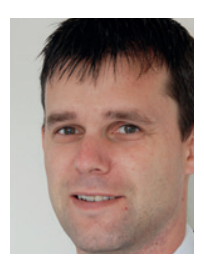

Dr. Matthias Gruber ist Oberarzt an der Medizinischen Klinik und Poliklinik III des Universitätsklinikums Carl Gustav Carus der TU Dresden matthias.gruber@uniklinikumdresden.de

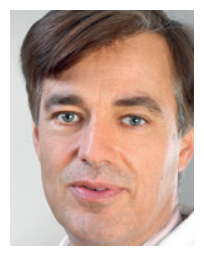

Prof. Dr. Stefan R. Bornstein ist Direktor der Medizinischen Klinik und Poliklinik III am Universitätsklinikum Carl Gustav Carus an der TU Dresden sowie Chair und Honorary Consultant für Diabetes und Endokrinologie am King's College London stefan.bornstein@uniklinikumdresden.de

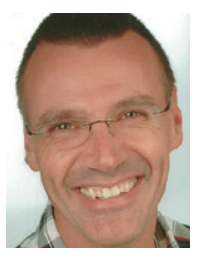

Prof. Dr. Andreas Barthel ist Standortleiter am Endokrinologikum Ruhr und assoziierter Mitarbeiter am Universitätsklinikum Carl Gustav Carus an der TU Dresden andreas.barthel@ endokrinologikum.com 
Abb. 1 a) Testikuläre adrenale Resttumoren (TART) bei einem klassischen adrenogenitalen Syndrom (Querschnitt durch beide Hoden): Nachweis bilateraler multipler echoarmer unregelmäßig konfigurierter Herde. b) Endokrin aktiver maligner Hodentumor (Längsschnitt): unilateraler, unifokal inhomogener, unregelmäßig konfigurierter und unscharf begrenzter Herd. Beide Tumore waren palpabel, der maligne Keimzelltumor derbe imponierend, die TART prallelastisch.
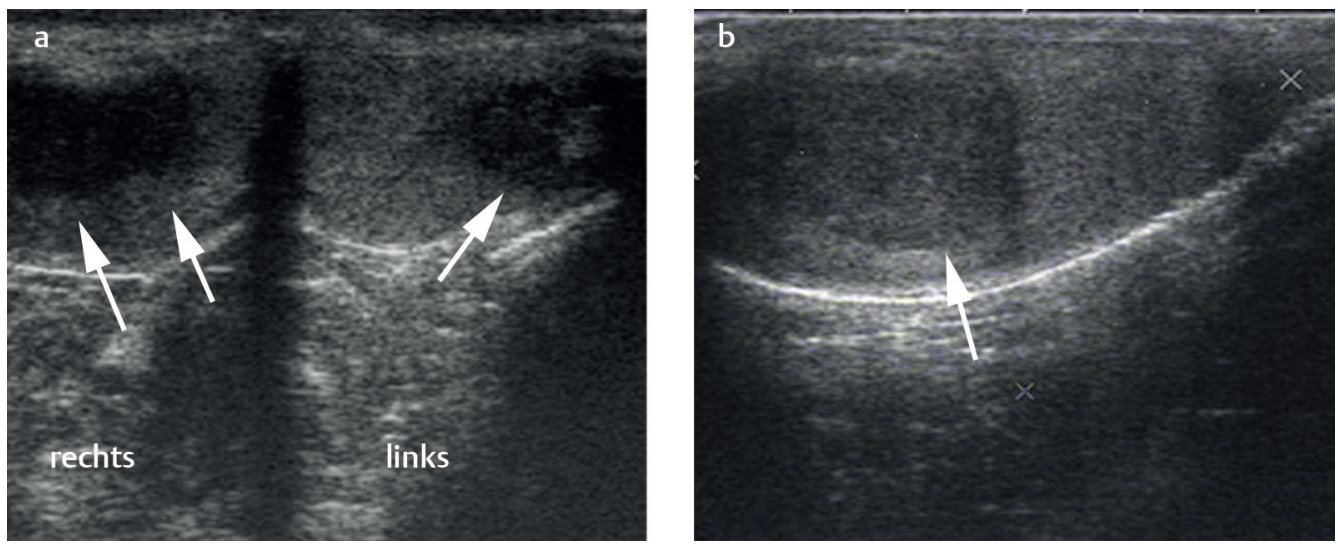

Der Befund wird z.B. erhoben bei der Abklärung von unerfülltem Kinderwunsch eines Paares, eines männlichen Hypogonadismus, eines auffälligen Tastbefundes der Hoden oder von Schmerzen oder aber als Zufallsbefund. Als Ursache wurde oft an eine unzureichende (biochemische) Kontrolle des AGS gedacht. Eine neuere Untersuchung mit longitudinaler Datenerhebung an der Universität München [13] fand jedoch keinen Beleg hierfür. Entscheidend ist wohl vor allem die Existenz von „adrenalem Restgewebe“ im Hoden und vielleicht auch die Schwere der Störung.

Wichtig ist die Differenzierung von Hodentumoren bei den typischerweise jungen Männern. In der Regel ist der TART-Befund bilateral ( $\bullet$ Abb. 1). Sofern noch nicht geschehen, muss die Diagnose des AGS gesichert werden - manchmal ist die Diagnose in der Transition in Vergessenheit geraten, unter Umständen ist diese Diagnose auch noch nicht bekannt. So fanden sich bei Familienuntersuchungen von 145 AGS-Kindern in der Generation der Eltern 10 bisher unbekannte (allerdings „nicht-klassische homozygote) AGS-Fälle [14].

\section{Literatur}

1 Tyler Miller R. Control of renal calcium, phosphate, electrolyte, and water excretion by the calcium-sensing receptor. Best Pract Res Clin Endocrinol Metab 2013; 27: 345-358

2 Brown EM Clinical lessons from the calcium-sensing receptor. Nat Clin Pract Endocrinol Metab 2007; 3 : 122-133

3 Nesbit MA, Hannan FM, Howles SA et al. Mutations affecting $G$-protein subunit $\alpha 11$ in hypercalcemia and hypocalcemia. N Engl J Med 2013; 368: 2476-2486

4 Nesbit MA, Hannan FM, Howles SA et al. Mutations in AP2S1 cause familial hypocalciuric hypercalcemia type 3. Nat Genet 2013; 45: 93-97

Interessenkonflikt SRB erklärt, dass er klinische Studien in Zusammenarbeit mit Novartis und Boehringer Ingelheim durchgeführt hat. $A B$, MG und $G B$ erklären, dass keine Interessenkonflikte bestehen.

DOI 10.1055/s-0041-100949 Dtsch Med Wochenschr 2015; 140: 470-472

(c) Georg Thieme Verlag KG . Stuttgart · New York . ISSN 0012-0472
Eine Operation ist indiziert bei therapierefraktären Schmerzen oder bei sonst nicht zu klärender Differenzierung gegenüber einem Malignom.

Es wird diskutiert, ob TART durch eine Intensivierung der Glukokortikoidtherapie beim AGS therapiert oder vermieden werden können. Eine Therapie mit höheren Glukokortikoiddosen ist aber problematisch und nach mehreren Studien ohne sichere Effekte [15]. Ideal wären bei der Betreuung männlicher AGS-Patienten serielle Ultraschalluntersuchungen der Hoden, um solche Veränderungen rechtzeitig zu erkennen.

\section{Klinische Relevanz}

Der sonographische Befund ist bei TART nur schwer von anderweitigen Hodentumoren abzugrenzen. Differenzialdiagnostisch wegweisend ist der charakteristische oftmals bilaterale und multifokale Herdbefund bei TART. Er sollte eine endokrinologische Abklärung und ggf. nachfolgende molekulargenetische Sicherung eines AGS zur Folge haben.

8 Dumitrescu AM, Refetoff S. The syndromes of reduced sensitivity to thyroid hormone. Biochim Biophys Acta 2013; 1830: 3987-4003

9 Visser T]. Thyroid hormone transporters and resistance. Endocr Dev 2013; 24: 1-10

10 van Mullem AA, Visser T], Peeters RP. Clinical Consequences of Mutations in Thyroid Hormone Receptor- $\alpha 1$. Eur Thyroid J 2014; 3: 17-24

11 Brent GA. Mechanisms of thyroid hormone action. I Clin Invest 2012; 122: 3035-3043

12 Merke DP, Bornstein SR. Congenital adrenal hyperplasia. Lancet 2005; 365: 2125-2136

13 Reisch N, Rottenkolber M, Greifenstein A et al. Testicular adrenal rest tumors develop independently of long-term disease control: a longitudinal analysis of 50 adult men with congenital adrenal hyperplasia due to classic 21-hydroxylase deficiency. J Clin Endocrinol Metab 2013; 98: E1820-E1826

14 Nandagopal R, Sinaii N, Avila NA et al. Phenotypic profiling of parents with cryptic nonclassic congenital adrenal hyperplasia: findings in 145 unrelated families. Eur J Endocrinol 2011; 164: 977-984

15 Ph DJ, Wojcik M, Tyrawa K et al. Testicular Adrenal Rest Tumors in Congenital Adrenal Hyperplasia-A Case Report and Literature Overview. Endocr Pract 2014; 20: e219-e224 